\title{
Differences of Operators of Lupaş Type
}

\author{
VIJAY GUPTA*
}

ABSTRACT. In the present article, we study the approximation of difference of operators and find the quantitative estimates for the difference of Lupaş operators with Lupaş-Szász operators and Lupaş-Kantorovich operators in terms of modulus of continuity. Also, we find the quantitative estimate for the difference of Lupaş-Kantorovich operators and Lupaş-Szász operators.

Keywords: Difference of operators, Lupaş operators, Lupaş-Szász operators, Lupaş-Kantorovich operators, Modulus of continuity.

2010 Mathematics Subject Classification: 41A25, 41A30.

\section{INTRODUCTION}

Approximation for linear positive operators to functions in real and complex setting is an active area of research amongst researchers. Several new operators have been constructed in last six decades and their approximation behaviours have been studied. Concerning approximation properties of linear positive operators the convergence is one of the important aspects, several methods and techniques have been applied to get the direct results in ordinary and simultaneous approximation, we mention some of the recent work viz. [1-4], [8-10], [12] etc.

Acu-Rasa [5] and Aral et al [7] established some interesting results for the difference of operators in order to generalize the problem posed by A. Lupaş [16] on polynomial differences. Some of the results on this topic are compiled in the recent book by Gupta et al [14].

Very recently the author in [11], provided a general result for the difference of operators and applied the result to Szász type operators. We consider here the Lupaş operators and its variants and find the quantitative estimates for the differences of such operators. A. Lupaş [16] proposed a discrete operators, which for $f \in C[0, \infty)$, are defined as

$$
L_{n}(f, x):=\sum_{k=0}^{\infty} l_{n, k}(x) F_{n, k}(f),
$$

where $F_{n, k}: D \rightarrow \mathbb{R}$ be positive linear functional defined on a subspace $D$ of $C[0, \infty)$ and

$$
l_{n, k}(x)=2^{-n x} \frac{(n x)_{k}}{k ! 2^{k}}, \quad F_{n, k}(f)=f\left(\frac{k}{n}\right)
$$

It was observed that these operators are linear and positive and preserve linear functions.

Received: August 13, 2018; In revised form: August 17, 2018; Accepted: August 19, 2018

*Corresponding author: V. Gupta; vijaygupta2001@hotmail.com

DOI: $10.33205 / \mathrm{cma} .452962$ 
Remark 1.1. For the Lupaş operators, we have $F_{n, k}(f)=f\left(\frac{k}{n}\right)$ such that $F_{n, k}\left(e_{0}\right)=1, b^{F_{n, k}}:=$ $F_{n, k}\left(e_{1}\right)$ If we denote $\mu_{r}^{F_{n, k}}=F_{n, k}\left(e_{1}-b^{F_{n, k}} e_{0}\right)^{r}, r \in \mathbb{N}$, then by simple computation, we have

$$
\begin{aligned}
\mu_{2}^{F_{n, k}} & :=F_{n, k}\left(e_{1}-b^{F_{n, k}} e_{0}\right)^{2}=0 \\
\mu_{4}^{F_{n, k}} & :=F_{n, k}\left(e_{1}-b^{F_{n, k}} e_{0}\right)^{4}=0
\end{aligned}
$$

Remark 1.2. The The moments of Lupaş operators with $e_{r}(t)=t^{r}, r \in \mathbb{N} \cup\{0\}$ are given by

$$
\begin{aligned}
& L_{n}\left(e_{0}, x\right)=1, \\
& L_{n}\left(e_{1}, x\right)=x, \\
& L_{n}\left(e_{2}, x\right)=x^{2}+\frac{2 x}{n}, \\
& L_{n}\left(e_{3}, x\right)=x^{3}+\frac{6 x^{2}}{n}+\frac{6 x}{n^{2}}, \\
& L_{n}\left(e_{4}, x\right)=x^{4}+\frac{12 x^{3}}{n}+\frac{36 x^{2}}{n^{2}}+\frac{26 x}{n^{3}}, \\
& L_{n}\left(e_{5}, x\right)=x^{5}+\frac{20 x^{4}}{n}+\frac{120 x^{3}}{n^{2}}+\frac{250 x^{2}}{n^{3}}+\frac{150 x}{n^{4}}, \\
& L_{n}\left(e_{6}, x\right)=x^{6}+\frac{30 x^{5}}{n}+\frac{300 x^{4}}{n^{2}}+\frac{1230 x^{3}}{n^{3}}+\frac{2040 x^{2}}{n^{4}}+\frac{1082 x}{n^{5}} .
\end{aligned}
$$

\section{DIFFERENCE OF OPERATORS}

Let $C_{B}[0, \infty)$ be the class of bounded continuous functions defined on the interval $[0, \infty)$ equipped with the norm $\|\|=.\sup _{x \in[0, \infty)}|f(x)|<\infty$. Let us consider another operator $V_{n}$ having the same Lupaş basis $l_{n, k}(x)$ such that

$$
V_{n}(f, x)=\sum_{k=0}^{\infty} l_{n, k}(x) G_{n, k}(f),
$$

where $G_{n, k}: D \rightarrow \mathbb{R}$. Following [11], we have the following quantitative general result.

Theorem 2.1. [11] Let $f^{(s)} \in C_{B}[0, \infty), s \in\{0,1,2\}$ and $x \in[0, \infty)$, then for $n \in \mathbb{N}$, we have

$$
\left|\left(L_{n}-V_{n}\right)(f, x)\right| \leq\left\|f^{\prime \prime}\right\| \alpha(x)+\omega\left(f^{\prime \prime}, \delta_{1}\right)(1+\alpha(x))+2 \omega\left(f, \delta_{2}(x)\right),
$$

where

$$
\alpha(x)=\frac{1}{2} \sum_{k=0}^{\infty} l_{n, k}(x)\left(\mu_{2}^{F_{n, k}}+\mu_{2}^{G_{n, k}}\right)
$$

and

$$
\delta_{1}^{2}=\frac{1}{2} \sum_{k=0}^{\infty} l_{n, k}(x)\left(\mu_{4}^{F_{n, k}}+\mu_{4}^{G_{n, k}}\right), \delta_{2}^{2}=\sum_{k=0}^{\infty} l_{n, k}(x)\left(b^{F_{n, k}}-b^{G_{n, k}}\right)^{2} .
$$

We now establish quantitative estimates for the difference of Lupaş operators with the LupaşKantorovich operators and Lupaş-Szász operators. 
2.1. Lupaş and Lupaş-Kantorovich operators. In [6] Agratini proposed the Kantorovich variant of the Lupaş operators as

$$
K_{n}(f, x):=\sum_{k=0}^{\infty} l_{n, k}(x) G_{n, k}(f)=n \sum_{k=0}^{\infty} l_{n, k}(x) \int_{\frac{k}{n}}^{\frac{k+1}{n}} f(t) d t
$$

where

$$
G_{n, k}(f)=n \int_{\frac{k}{n}}^{\frac{k+1}{n}} f(t) d t
$$

Below, we present the quantitative estimate for difference of Lupaş and Lupaş-Kantorovich operators.

Theorem 2.2. Let $f^{(s)} \in C_{B}[0, \infty), s \in\{0,1,2\}$ and $x \in[0, \infty)$, then for $n \in \mathbb{N}$, we have

$$
\left|\left(K_{n}-L_{n}\right)(f, x)\right| \leq \frac{1}{24 n^{2}}\left\|f^{\prime \prime}\right\|+\omega\left(f^{\prime \prime}, \frac{1}{4 \sqrt{10} n^{2}}\right)\left(1+\frac{1}{24 n^{2}}\right)+2 \omega\left(f, \frac{1}{2 n}\right) .
$$

Proof. Following Theorem 2.1, by simple computation, we have

and

$$
b^{G_{n, k}}=G_{n, k}\left(e_{1}\right)=\frac{2 k+1}{2 n}
$$

$$
\begin{aligned}
\mu_{2}^{G_{n, k}} & :=G_{n, k}\left(e_{1}-b^{G_{n, k}} e_{0}\right)^{2} \\
& =G_{n, k}\left(e_{2}\right)+\left(\frac{2 k+1}{2 n}\right)^{2}-2 G_{n, k}\left(e_{1}\right)\left(\frac{2 k+1}{2 n}\right) \\
& =\frac{3 k^{2}+3 k+1}{3 n^{2}}-\left(\frac{2 k+1}{2 n}\right)^{2} \\
& =\frac{1}{12 n^{2}} .
\end{aligned}
$$

Next, using Remark 1.2, we have

$$
\alpha(x):=\frac{1}{2} \sum_{k=0}^{\infty} l_{n, k}(x)\left(\mu_{2}^{F_{n, k}}+\mu_{2}^{G_{n, k}}\right)=\frac{1}{24 n^{2}} .
$$

Further,

$$
\begin{aligned}
\mu_{4}^{G_{n, k}} & :=G_{n, k}\left(e_{1}-b^{G_{n, k}} e_{0}\right)^{4} \\
& =G_{n, k}\left(e_{4}\right)-4 G_{n, k}\left(e_{3}\right)\left(\frac{2 k+1}{2 n}\right)+6 G_{n, k}\left(e_{2}\right)\left(\frac{2 k+1}{2 n}\right)^{2} \\
& -4 G_{n, k}\left(e_{1}\right)\left(\frac{2 k+1}{2 n}\right)^{3}+G_{n, k}\left(e_{0}\right)\left(\frac{2 k+1}{2 n}\right)^{4} \\
& =\frac{5 k^{4}+10 k^{3}+10 k^{2}+5 k+1}{5 n^{4}}-4 \frac{4 k^{3}+6 k^{2}+4 k+1}{4 n^{3}}\left(\frac{2 k+1}{2 n}\right) \\
& +6 \frac{3 k^{2}+3 k+1}{3 n^{2}}\left(\frac{2 k+1}{2 n}\right)^{2}-3\left(\frac{2 k+1}{2 n}\right)^{4}=\frac{1}{80 n^{4}} .
\end{aligned}
$$


Then using Remark 1.1 and above equality, we get

$$
\delta_{1}^{2}(x)=\frac{1}{2} \sum_{k=0}^{\infty} l_{n, k}(x)\left(\mu_{4}^{F_{n, k}}+\mu_{4}^{G_{n, k}}\right)=\frac{1}{160 n^{4}} .
$$

and by using Remark 1.2, we have

$$
\begin{aligned}
\delta_{2}^{2}(x) & =\sum_{k=0}^{\infty} l_{n, k}(x)\left(b^{F_{n, k}}-b^{G_{n, k}}\right)^{2} \\
& =\sum_{k=0}^{\infty} l_{n, k}(x)\left[\frac{k}{n}-\frac{2 k+1}{2 n}\right]^{2} \\
& =\frac{1}{4 n^{2}} .
\end{aligned}
$$

This completes the proof of the theorem.

2.2. Lupaş and Lupaş-Szász operators. The Lupaş-Szász operators are defined as

$$
S_{n}(f ; x)=n \sum_{k=1}^{\infty} l_{n, k}(x) \int_{0}^{\infty} s_{n, k-1}(t) f(t) d t+l_{n, 0}(x) f(0),
$$

where the Szász basis function is defined as $s_{n, k}(t)=\frac{e^{-n t}(n t)^{k}}{k !}$.

If we denote

$$
H_{n, k}(f)=n \int_{0}^{\infty} s_{n, k-1}(t) f(t) d t, 0 \leq k<\infty, H_{n, 0}(f)=f(0)
$$

then the operators (2.3) take the following form:

$$
S_{n}(f, x)=\sum_{k=0}^{\infty} l_{n, k}(x) H_{n, k}(f) .
$$

We present below the quantitative estimate for difference of Lupaş and Lupaş-Szász operators.

Theorem 2.3. Let $f^{(s)} \in C_{B}[0, \infty), s \in\{0,1,2\}$ and $x \in[0, \infty)$, then for $n \in \mathbb{N}$, we have

$$
\left|\left(S_{n}-L_{n}\right)(f, x)\right|=\left\|f^{\prime \prime}\right\| \frac{x}{2 n}+\omega\left(f^{\prime \prime}, \sqrt{\frac{3 x^{2}}{2 n^{2}}+\frac{6 x}{n^{3}}}\right)\left(1+\frac{x}{2 n}\right) .
$$

Proof. By simple computation, we have

$$
b^{H_{n, k}}=H_{n, k}\left(e_{1}\right)=\frac{k}{n} .
$$

Also, we have

$$
\begin{aligned}
\mu_{2}^{H_{n, k}} & :=H_{n, k}\left(e_{1}-b^{H_{n, k}} e_{0}\right)^{2} \\
& =H_{n, k}\left(e_{2}\right)+\left(\frac{k}{n}\right)^{2}-2 H_{n, k}\left(e_{1}\right)\left(\frac{k}{n}\right) \\
& =\frac{k(k+1)}{n^{2}}-\frac{k^{2}}{n^{2}}=\frac{k}{n^{2}} .
\end{aligned}
$$


Next, using Remark 1.1, we have

$$
\alpha(x):=\frac{1}{2} \sum_{k=0}^{\infty} l_{n, k}(x)\left(\mu_{2}^{F_{n, k}}+\mu_{2}^{H_{n, k}}\right)=\frac{x}{2 n} .
$$

and

$$
\begin{aligned}
\mu_{4}^{H_{n, k}} & :=H_{n, k}\left(e_{1}-b^{H_{n, k}} e_{0}\right)^{4} \\
& =H_{n, k}\left(e_{4}\right)-4 H_{n, k}\left(e_{3}\right)\left(\frac{k}{n}\right)+6 H_{n, k}\left(e_{2}\right)\left(\frac{k}{n}\right)^{2} \\
& -4 H_{n, k}\left(e_{1}\right)\left(\frac{k}{n}\right)^{3}+H_{n, k}\left(e_{0}\right)\left(\frac{k}{n}\right)^{4} \\
& =\frac{3 k^{2}+6 k}{n^{4}} .
\end{aligned}
$$

Then by Remark 1.1, we have

$$
\begin{aligned}
\delta_{1}^{2}(x) & =\frac{1}{2} \sum_{k=0}^{\infty} l_{n, k}(x)\left(\mu_{4}^{F_{n, k}}+\mu_{4}^{H_{n, k}}\right) \\
& =\sum_{k=0}^{\infty} l_{n, k}(x) \frac{3 k^{2}+6 k}{2 n^{4}} \\
& =\frac{3 x^{2}}{2 n^{2}}+\frac{6 x}{n^{3}} .
\end{aligned}
$$

and by using above identities, we have

$$
\delta_{2}^{2}(x)=\sum_{k=0}^{\infty} l_{n, k}(x)\left(b^{F_{n, k}}-b^{H_{n, k}}\right)^{2}=0 .
$$

This completes the proof of the theorem.

\subsection{Lupaş-Kantorovich and Lupaş-Szász operators.}

Theorem 2.4. Let $f^{(s)} \in C_{B}[0, \infty), s \in\{0,1,2\}$ and $x \in[0, \infty)$, then for $n \in \mathbb{N}$, we have

$$
\begin{aligned}
\left|\left(S_{n}-K_{n}\right)(f, x)\right| & =\left\|f^{\prime \prime}\right\|\left(\frac{1}{24 n^{2}}+\frac{x}{2 n}\right)+2 \omega\left(f, \frac{1}{2 n}\right) \\
& +\omega\left(f^{\prime \prime}, \sqrt{\frac{1}{160 n^{4}}+\frac{3 x^{2}}{2 n^{2}}+\frac{6 x}{n^{3}}}\right)\left(1+\frac{1}{24 n^{2}}+\frac{x}{2 n}\right) .
\end{aligned}
$$

Proof. By previous subsections, we have

$$
\begin{aligned}
b^{G_{n, k}}=G_{n, k}\left(e_{1}\right) & =\frac{2 k+1}{2 n}, \quad b^{H_{n, k}}=H_{n, k}\left(e_{1}\right)=\frac{k}{n} . \\
\mu_{2}^{G_{n, k}} & =\frac{1}{12 n^{2}}, \quad \mu_{2}^{H_{n, k}}=\frac{k}{n^{2}}
\end{aligned}
$$

and

$$
\mu_{4}^{G_{n, k}}=\frac{1}{80 n^{4}}, \quad \mu_{2}^{H_{n, k}}=\frac{3 k^{2}+6 k}{n^{4}} .
$$


Thus, we have

$$
\begin{aligned}
\alpha(x) & :=\frac{1}{2} \sum_{k=0}^{\infty} l_{n, k}(x)\left(\mu_{2}^{G_{n, k}}+\mu_{2}^{H_{n, k}}\right)=\frac{1}{24 n^{2}}+\frac{x}{2 n} \\
\delta_{1}^{2}(x) & =\frac{1}{2} \sum_{k=0}^{\infty} l_{n, k}(x)\left(\mu_{4}^{G_{n, k}}+\mu_{4}^{H_{n, k}}\right) \\
& =\sum_{k=0}^{\infty} l_{n, k}(x)\left[\frac{1}{160 n^{4}}+\frac{3 k^{2}+6 k}{2 n^{4}}\right] \\
& =\frac{1}{160 n^{4}}+\frac{3 x^{2}}{2 n^{2}}+\frac{6 x}{n^{3}} .
\end{aligned}
$$

and by using above identities, we have

$$
\delta_{2}^{2}(x)=\sum_{k=0}^{\infty} l_{n, k}(x)\left(b^{G_{n, k}}-b^{H_{n, k}}\right)^{2}=\frac{1}{4 n^{2}} .
$$

The result follows by combining above estimates as in Theorem 2.1.

Remark 2.3. In [13] Gupta et al and [15] Gupta-Yadav also considered Lupaş-Beta type operators, the difference estimates can be obtained analogously, the analysis is different we can discuss them elsewhere.

\title{
REFERENCES
}

[1] T. Acar: Asymptotic formulas for generalized Szász--Mirakyan operators, Appl. Math. Comput. 263 (2015), $233-239$.

[2] T. Acar, V. Gupta and A. Aral: Rate of convergence for generalized Szász operators, Bull. Math. Sci. 1 (1)(2011), 99-113.

[3] R. P. Agarwal and V. Gupta: On q-analogue of a complex summation-integral type operators in compact disks, J. Inequal. Appl. 2012 (1) (2012), Art. 111.

[4] A. Aral, V. Gupta and R. P. Agarwal: Applications of $q$ Calculus in Operator Theory, Springer-Verlag, New York, 2013.

[5] A. M. Acu and I. Rasa: New estimates for the differences of positive linear operators, Numer. Algorithms 73(2016), $775-789$.

[6] O. Agratini: On a sequence of linear and positive operators, Facta Univ. (NIS) Ser. Math. Inform. 14 (1999), $41--48$.

[7] A. Aral, D. Inoan and I. Rasa: On differences of linear positive operators, Anal. Math. Phys.(2018). DOI https://doi.org/10.1007/s1332

[8] M. Bodur, O. G. Y1lmaz and A. Aral: Approximation by Baskakov-Szász-Stancu operators preserving exponential functions, Const. Math. Anal. 1 (1) (2018), 1-8.

[9] N. Deo: Faster rate of convergence on Srivastava-Gupta operators, Appl. Math. Comput. 218 (21)(2012), 10486-10491.

[10] S. G. Gal and V. Gupta: Quantitative estimates for a new complex Durrmeyer operator in compact disks, Appl. Math. Comput. 218 (6) (2011), 2944-2951.

[11] V. Gupta: On difference of operators with applications to Szász type operators, communicated.

[12] V. Gupta: An estimate on the convergence of Baskakov-Bézier operators, J. Math. Anal. Appl. 312 (1)(2005), $280-288$.

[13] V. Gupta, Th. M. Rassias and R. Yadav: Approximation by Lupaş-Beta integral operators, Appl. Math. Comput. 236 (2014), 19-26.

[14] V. Gupta, T. M. Rassias, P. N. Agrawal and A. M. Acu: Recent Advances in Constructive Approximation Theory, Springer Optimization and Its Applications, vol. 138, (2018), Springer, Cham.

[15] V. Gupta and R. Yadav: On approximation of certain integral operators, Acta Math. Vietnam. 39 (2)(2014), $193-203$.

[16] A. Lupaş: The approximation by means of some linear positive operators, In: Approximation Theory (M.W. Müller others, eds), pp. 201-227. Akademie-Verlag, Berlin (1995).

\author{
Netaji Subhas InStitute of TECHNOLOGy \\ DEPARTMENT OF MATHEMATICS \\ SECTOR 3 DWARKA, NEW DELHI 110078, INDIA \\ E-mail address: vi jaygupta2001@hotmail.com
}

